\title{
ON THE LOCALLY ANTISIMPLE RADICAL
}

\section{PATRICK N. STEWART $\dagger$}

(Received 9 March, 1970)

In this paper we presuppose a familiarity with the terminology and the basic results of radical theory, all of which can be found in Divinsky [2].

Andrunakievic's antisimple radical class $\beta_{\phi}$ (see [1] and [2]) is the upper radical class determined by the class of all subdirectly irreducible rings with idempotent hearts (the heart of a ring is the intersection of all the nonzero ideals). A ring $R$ is in $\beta_{\phi}$ if and only if $R$ cannot be homomorphically mapped onto a subdirectly irreducible ring with an idempotent heart. This radical class is a special radical class; that is, the class $\beta_{\phi}$ is hereditary, contains all nilpotent rings, and every $\beta_{\phi}$ semisimple ring is isomorphic to a subdirect sum of prime $\beta_{\phi}$ semisimple rings. Levitzki's locally nilpotent radical class $\mathscr{L}$ (the class of all rings $R$ such that every finitely generated subring of $R$ is nilpotent) is strictly contained in $\beta_{\phi}$, which is in turn strictly contained in the Brown-McCoy radical class $\mathscr{G}$ (i.e., $\mathscr{L}<\beta_{\phi}<\mathscr{G}$ ).

The main results of this paper are that the class $\beta_{\phi}^{*}$ of all locally $\beta_{\phi}$ rings (rings such that every finitely generated subring is in $\beta_{\phi}$ ) is a special radical class and that $\mathscr{L} \leqq \beta_{\phi}^{*} \leqq \mathcal{N}$, where $\mathcal{N}$ is the special radical class of all nil rings.

To prove that $\beta_{\phi}^{*}$ is a radical class we require the following result about simple idempotent rings.

PROPOSITION 1. If $S$ is a nonzero simple idempotent ring, then there is a finitely generated subring of $S$ which can be homomorphically mapped onto a subdirectly irreducible ring with a simple idempotent heart.

Proof. Let $S$ be a nonzero simple idempotent ring. By Theorem 55 in Divinsky [2], $S \notin \mathscr{L}$ and so, by Theorem 53 in Divinsky [2], there is an element $x \in S$ such that $x^{4} \neq 0$. Then $S x^{2} S$ is a nonzero ideal of $S$; so $S=S x^{2} S$. Thus there are elements $r_{1}, \ldots, r_{k}$ and $s_{1}, \ldots, s_{k}$ of $S$ such that

$$
x=\sum_{i=1}^{k} r_{i} x^{2} s_{i} .
$$

Let $S^{\prime}$ be the subring of $S$ that is generated by the set $\left\{r_{1}, \ldots, r_{k}, x, s_{1}, \ldots, s_{k}\right\} . \quad$ By Zorn's Lemma we can choose a maximal ideal $I$ in the set of all ideals of $S^{\prime}$ that do not contain $x$. Then $S^{\prime} / I$ is subdirectly irreducible with heart $H=(X+I) / I$, where $X$ is the ideal of $S^{\prime}$ that is generated by $x$. If $x^{2} \in I$, then, by $\left(^{*}\right), x \in I$ and so, since $x \notin I, x^{2} \notin I$. Therefore $H^{2} \neq(0)$ and thus, since the heart of a subdirectly irreducible ring must be either a zero ring or a simple idempotent ring (see Lemma 75 in Divinsky [2]), $H$ is a simple idempotent ring. This completes the proof.

An interesting conclusion which follows from this proposition is that, if there is a simple idempotent nil ring, then there is one which is the heart of a finitely generated nil ring and so

$\dagger$ Most of the results in this paper appeared in the author's doctoral thesis, which was written under the supervision of Professor N. J. Divinsky. 
is certainly countable. This seems to support the long standing conjecture that there are no nontrivial simple idempotent nil rings.

From the proposition we may also conclude that $\beta_{\phi}^{*} \leqq \beta_{\phi}$. To see this, let $R$ be a ring which is not in $\beta_{\phi}$. Then $R$ can be homomorphically mapped onto a subdirectly irreducible ring with idempotent heart $S$. The proposition states that there is a finitely generated subring of $S$ which can also be homomorphically mapped onto such a ring. This subring of $S$ must be a homomorphic image of some finitely generated subring of $R$ and so $R \notin \beta_{\phi}^{*}$, because this finitely generated subring of $R$ is not in $\beta_{\phi}$.

THEOREM 1. The class $\beta_{\phi}^{*}$ is a radical class.

Proof. It is sufficient to establish the following:

(a) The class $\beta_{\phi}^{*}$ is homomorphically closed.

(b) If $B$ is an ideal of a ring $A$ and both $A / B$ and $B$ are in $\beta_{\phi}^{*}$, then $A \in \beta_{\phi}^{*}$.

(c) For any ring $R$, the sum of all the ideals of $R$ that (as rings) are in $\beta_{\phi}^{*}$ is also in $\beta_{\phi}^{*}$. closed.

Since the class $\beta_{\phi}$ is homomorphically closed, it follows that $\beta_{\phi}^{*}$ is also homomorphically

Assume that $B$ is an ideal of a ring $A$ and that both $A / B$ and $B$ are in $\beta_{\phi}^{*}$. Let $A^{\prime}$ be a finitely generated subring of $A$. Then

$$
\frac{A^{\prime}+B}{B} \cong \frac{A^{\prime}}{A^{\prime} \cap B}
$$

and, since $A / B \in \beta_{\phi}^{*},\left(A^{\prime}+B\right) / B \in \beta_{\phi}$. Now $A^{\prime} \cap B \subseteq B \in \beta_{\phi}^{*}$; so $A^{\prime} \cap B \in \beta_{\phi}^{*} \leqq \beta_{\phi}$. Thus both $A^{\prime} /\left(A^{\prime} \cap B\right)$ and $A^{\prime} \cap B$ are in $\beta_{\phi}$; so $A^{\prime} \in \beta_{\phi}\left(\beta_{\phi}\right.$ is a radical class and is therefore closed under extensions). Hence $A \in \beta_{\phi}^{*}$ and so condition (b) is satisfied.

Let $R$ be a ring and let $I$ be the sum of all the $\beta_{\phi}^{*}$ ideals of $R$. If $R^{\prime}$ is a finitely generated subring of $I$, then $R^{\prime}$ is contained in the sum of finitely many $\beta_{\phi}^{*}$ ideals. So in order to establish (c) it is sufficient to show that a finite sum of $\beta_{\phi}^{*}$ ideals is again a $\beta_{\phi}^{*}$ ideal. Let $I_{1}$ and $I_{2}$ be $\beta_{\phi}^{*}$ ideals of $R$. Then

$$
\frac{I_{1}+I_{2}}{I_{2}} \cong \frac{I_{1}}{I_{1} \cap I_{2}}
$$

so $\left(I_{1}+I_{2}\right) / I_{2} \in \beta_{\phi}^{*}$ by (a). Hence, by (b), $I_{1}+I_{2} \in \beta_{\phi}^{*}$. The extension to any finite sum of ideals is obvious.

From (a), (b) and (c) it follows that any homomorphic image of a $\beta_{\phi}^{*}$ ring is a $\beta_{\phi}^{*}$ ring, that every ring $R$ contains a unique maximal $\beta_{\phi}^{*}$ ideal $\beta_{\phi}^{*}(R)$, and that $\beta_{\phi}^{*}\left(R / \beta_{\phi}^{*}(R)\right)=(0)$. Therefore $\beta_{\phi}^{*}$ is a radical class.

Since a nilpotent ring cannot be homomorphically mapped onto a ring with a nontrivial idempotent heart, $\mathscr{L} \leqq \beta_{\phi}^{*}$ and of course $\beta_{\phi}^{*}$ contains all nilpotent rings. The radical class $\beta_{\phi}^{*}$ is hereditary; in fact, subrings of rings in $\beta_{\phi}^{*}$ are in $\beta_{\phi}^{*}$. Thus the following theorem implies that $\beta_{\phi}^{*}$ is a special radical class.

THEOREM 2. A ring $R$ is $\beta_{\phi}^{*}$ semisimple if and only if $R$ is a subdirect sum of prime $\beta_{\phi}^{*}$ semisimple rings. 
Proof. Since subdirect sums of semisimple rings are semisimple, one direction is clear.

Conversely, let $R$ be a $\beta_{\phi}^{*}$ semisimple ring. It is sufficient to prove that for each nonzero $x \in R$ there is an ideal $I(x)$ of $R$ such that $x \notin I(x)$ and $R / I(x)$ is a prime $\beta_{\phi}^{*}$ semisimple ring.

Let $x$ be a nonzero element of $R$ and let $X$ be the ideal of $R$ that is generated by $x$. Since $X \notin \beta_{\phi}^{*}$, there is a finitely generated subring $R^{\prime} \subseteq X$ and an ideal $I^{\prime}$ of $R^{\prime}$ such that $R^{\prime} / I^{\prime}$ contains the nonzero simple idempotent heart $S^{\prime} / I^{\prime}$.

Let $Z$ be the set of all ideals $J$ of $R$ such that $R^{\prime} \cap J \subseteq I^{\prime}$. Clearly the union of an ascending chain of ideals in $Z$ is in $Z$; so by Zorn's Lemma we may choose $I(x)$ maximal in $Z$.

First we shall prove that $R / I(x)$ is $\beta_{\phi}^{*}$ semisimple. Let $L / I(x)$ be a nonzero ideal of $R / I(x)$. Now

$$
\frac{R^{\prime} \cap L+I(x)}{I(x)} \cong \frac{R^{\prime} \cap L}{R^{\prime} \cap I(x)}
$$

and $\left(R^{\prime} \cap L\right) /\left(R^{\prime} \cap I(x)\right)$ can be homomorphically mapped onto $\left(R^{\prime} \cap L+I^{\prime}\right) / I^{\prime}$, since $I(x) \in Z$. The ideal $L$ properly contains $I(x)$; so $R^{\prime} \cap L \notin I^{\prime}$. Thus $R^{\prime} \cap L+I^{\prime}$ is an ideal of $R^{\prime}$ that properly contains $I^{\prime}$. Since $S^{\prime} / I^{\prime}$ is a simple ring, $S^{\prime} \subseteq R^{\prime} \cap L+I^{\prime}$ and so $S^{\prime} \mid I^{\prime} \subseteq$ $\left(R^{\prime} \cap L+I^{\prime}\right) / I^{\prime}$. It follows that $\left(R^{\prime} \cap L+I^{\prime}\right) / I^{\prime}$ is subdirectly irreducible with idempotent heart $S^{\prime} \mid I^{\prime}$. Thus $\left(R^{\prime} \cap L+I^{\prime}\right) / I^{\prime}$ is not in $\beta_{\phi}$ and, since $\beta_{\phi}^{*} \leqq \beta_{\phi},\left(R^{\prime} \cap L+I^{\prime}\right) / I^{\prime} \notin \beta_{\phi}^{*}$. Therefore $\left(R^{\prime} \cap L+I(x)\right) / I(x)$ and hence $L / I(x)$ is not in $\beta_{\phi}^{*}$. It follows that $R / I(x)$ is $\beta_{\phi}^{*}$ semisimple.

Now we shall prove that $R / I(x)$ is a prime ring. Suppose that $L$ and $H$ are two ideals of $R$ that properly contain $I(x)$. As above, the maximality of $I(x)$ implies that $R^{\prime} \cap L+I^{\prime} \supseteq S^{\prime}$ and $R^{\prime} \cap H+I^{\prime} \supseteq S^{\prime}$. If $L H \subseteq I(x)$ we must have that

$$
\frac{S^{\prime}}{I^{\prime}}=\left(\frac{S^{\prime}}{I^{\prime}}\right)^{2} \subseteq \frac{\left(R^{\prime} \cap L+I^{\prime}\right)\left(R^{\prime} \cap H+I^{\prime}\right)+I^{\prime}}{I^{\prime}} \subseteq \frac{R^{\prime} \cap I(x)+I^{\prime}}{I^{\prime}} .
$$

This implies that $S^{\prime} \subseteq R^{\prime} \cap I(x)+I^{\prime} \subseteq I^{\prime}$, which is a contradiction. Thus $H L \nsubseteq I(x)$; so $R / I(x)$ is a prime ring.

Therefore $R / I(x)$ is a prime $\beta_{\phi}^{*}$ semisimple ring and, since $R^{\prime} \nsubseteq I(x), x \notin I(x)$.

It follows from the theorem that, for any ring $R, \beta_{\phi}^{*}(R)$ can be represented as an intersection of prime ideals $P_{\alpha}(\alpha \in \Lambda)$ such that $R / P_{\alpha}$ is $\beta_{\phi}^{*}$ semisimple for each $\alpha \in \Lambda$. For those rings $R$ such that $R / \beta_{\phi}^{*}(R)$ is a generalized right Goldie ring (see [5]), Michler's Theorem 1 in [5] implies that $\beta_{\phi}^{*}(R)$ can be represented as the intersection of all the maximal $\beta_{\phi}^{*}$ subrings of $R$. Michler's Theorem may be similarly applied to the radical class $\beta_{\phi} \cap \mathcal{N}$ which is discussed below.

For rings $R$ satisfying the ascending chain condition on right annihilators it is well-known that $\mathscr{L}(R)=\mathcal{N}(R)$. In the following proposition we shall prove that $\mathscr{L} \leqq \beta_{\phi}^{*} \leqq \beta_{\phi} \cap \mathcal{N} \leqq \mathcal{N}$, so that for rings satisfying the ascending chain condition on right annihilators all four of these radicals are equal.

Three unsolved questions concerning the class $\beta_{\phi}^{*}$ are:

(1) Does the $\beta_{\phi}^{*}$ radical of a ring $R$ contain all the one-sided $\beta_{\phi}^{*}$ ideals of $R$ ?

(2) Is $\beta_{\phi}^{*}<\mathscr{N}$ ?

(3) Is $\mathscr{L}<\beta_{\phi}^{*}$ ? 
In Proposition 2 we shall prove that (2) is equivalent to the question of the existence of a nontrivial simple idempotent nil ring. On the other hand, if the answer to (3) is in the affirmative, it should be possible to show this by example without becoming involved in the idempotent nil ring problem. We are able to show that the related class of rings $\beta_{\phi} \cap \mathcal{N}$ does strictly contain $\mathscr{L}$. This class of rings is a special radical class; indeed, it is straightforward to verify that the intersection of any collection of (special) radical classes is a (special) radical class.

In the following proposition we collect some results about the relationships between the special radicals $\mathscr{L}, \beta_{\phi}^{*}, \beta_{\phi} \cap \mathcal{N}$ and $\mathcal{N}$.

Proposition 2.

(a) $\mathscr{L} \leqq \beta_{\phi}^{*} \leqq \beta_{\phi} \cap \mathcal{N} \leqq \mathcal{N}$.

(b) The following are equivalent:

(i) $\beta_{\phi} \cap \mathcal{N}<\mathcal{N}$

(ii) $\beta_{\phi}^{*}<\mathcal{N}$.

(iii) There is a nontrivial simple idempotent nil ring.

(c) $\mathscr{L}<\beta_{\phi} \cap \mathcal{A}^{\prime}$.

Proof. (a) We have already seen that $\mathscr{L} \leqq \beta_{\phi}^{*} \leqq \beta_{\phi}$. In order to establish (a) it is sufficient to prove that $\beta_{\phi}^{*} \leqq \mathcal{N}$.

Let $R \in \beta_{\phi}^{*}$ and $x \in R$. Then the subring $\langle x\rangle$ that is generated by $x$ is in $\beta_{\phi}$. Now $\beta_{\phi}<\mathscr{G}$ and a commutative ring is in $\mathscr{G}$ if and only if it is in the Jacobson radical class $J$ (see Divinsky [2, Lemma 89]). It follows that $\langle x\rangle \in J$.

In [3] Goldman proves that, if $R$ is a commutative Hilbert ring (A ring $R$ is a Hilbert ring if and only if $J(\bar{R})=\mathscr{N}(\bar{R})$ for all homomorphic images $\bar{R}$ of $R$ ), then so is the polynomial ring $R[X]$. Clearly the ring $Z$ of integers is a Hilbert ring; so Goldman's theorem implies that $Z[X]$ is a Hilbert ring. Now one easily sees that the ideal of $Z[X]$ that is generated by $X$ is also a Hilbert ring. The ring $\langle x\rangle$ is a homomorphic image of this ideal; so $J(\langle x\rangle)=$ $\mathscr{N}(\langle x\rangle)$. Since $\langle x\rangle \in J,\langle x\rangle \in \mathscr{N}$.

We have shown that, if $R \in \beta_{\phi}^{*}$ and $x \in R$, then $\langle x\rangle \in \mathscr{N}$. Thus $R \in \mathscr{N}$ and so $\beta_{\phi}^{*} \leqq \mathscr{N}$.

(b) The proof is cyclic. Since $\beta_{\phi}^{*} \leqq \beta_{\phi} \cap \mathcal{N}$ it is clear that (i) implies (ii). Assume that $\beta_{\phi}^{*}<\mathscr{N}$. Then there exists a ring $R \in \mathcal{N}$ such that $R \notin \beta_{\phi}^{*}$; so some homomorphic image of a finitely generated subring of $R$ contains a nontrivial heart which is a simple idempotent nil ring. Finally to see that (iii) implies (i) we need only notice that any nontrivial simple idempotent nil ring is in $\mathscr{N}$ but not in $\beta_{\phi} \cap \mathcal{N}$.

(c) Let $R$ be any infinite dimensional, finitely generated nil algebra over a finite field $F$. Such rings were shown to exist by Golod and Shafarevitch [4]. Notice that $R$ is finitely generated as a ring.

Suppose that $I_{\alpha}(\alpha \in \Lambda)$ is an ascending chain of ideals of $R$ and that $R / I_{\alpha}$ is infinite dimensional over $F$ for all $\alpha \in \Lambda$. Let $I=\bigcup\left\{I_{\alpha}: \alpha \in \Lambda\right\}$. If $R / I$ is finite dimensional over $F$, then $R^{N} \subseteq I$ for some positive integer $N$ (since the nil radical of a finite dimensional algebra is nilpotent). However, since $R$ is a finitely generated ring, $R^{N}$ is a finitely generated ring; so $R^{N} \subseteq I_{\beta}$ for some $\beta \in \Lambda$. This contradicts the infinite dimensionality of $R / I_{\beta}$; so $R / I$ must be 
infinite dimensional over $F$. Thus we may choose, by Zorn's Lemma, an ideal $I$ of $R$ which is maximal in the set of all ideals $J$ of $R$ such that $R / J$ is infinite dimensional over $F$.

No proper homomorphic image of $R / I$ can be subdirectly irreducible with an idempotent heart, because all such homomorphic images are finite dimensional over $F$ and hence nilpotent. Suppose that $R / I$ itself is subdirectly irreducible with heart $S / I$. Then $R^{N} \subseteq S$ for some positive integer $N$ because $R / S$ is a finite dimensional nil ring. If $S / I$ is idempotent, then $(S / I)^{2}=$ $(S / I)^{N} \subseteq(R / I)^{N} \subseteq S / I$; so $(R / I)^{N}=S / I$. But then $S / I$ is a finitely generated idempotent nil ring. This contradicts Nakayama's Lemma; so $S / I$ is not idempotent. It follows that $R / I \in \beta_{\phi} \cap \mathcal{N}$. However, since $R / I$ is infinite dimensional over $F$ and $R / I$ is finitely generated as a ring, $R / I \notin \mathscr{L}$.

Added in proof. Yu. M. Ryabukhin has also obtained Proposition 1 for simple idempotent nil rings in his paper "Concerning the problem of the existence of simple nil rings ", Sib. Matem. Zh. 10 (1969), 950-956 (English translation: Siberian Math. J. 10 (1969), 698-702).

\section{REFERENCES}

1. V. Andrunakievic, Radicals in associative rings I, Mat. Sb. 44 (1958), 179-212.

2. N. J. Divinsky, Rings and radicals (Toronto, 1965).

3. O. Goldman, Hilbert rings and the Hilbert Nullstellensatz, Math. Zeit. 54 (1951), 136-140.

4. E. S. Golod and I. R. Shafarevitch, On towers of class fields, Izv. Akad. Nauk SSSR., Ser. Mat. 28 (1964), 261-272. 89-101.

5. G. Michler, Maximal nilpotent subrings of right noetherian rings, Glasgow Math. J. 8 (1967),

UNIVERSITY OF GLASGOW

GlasGow, W.2 